\title{
Epidemiological Profile and Therapeutics Results of Bladder Carcinoma in Women
}

\author{
Maryam Zaouit*, Hassan Jouhadi, Tarik Chekrine, Zineb Bouchbika, Nadia Benchekroun, \\ Nezha Tawfiq, Souha Sahraoui, Aabdellatif Benider
}

Mohamed Center VI for Cancer Treatment, CHU Ibn Rochd, Casablanca, Morocco

Email: ^meryem.zaouit@gmail.com

How to cite this paper: Zaouit, M., Jouhadi, H., Chekrine, T., Bouchbika, Z., Benchekroun, N., Tawfiq, N., Sahraoui, S. and Benider, A. (2022) Epidemiological Profile and Therapeutics Results of Bladder Carcinoma in Women. Open Journal of Epidemiology, 12, 68-74.

https://doi.org/10.4236/ojepi.2022.121006

Received: January 12, 2022

Accepted: February 14, 2022

Published: February 17, 2022

Copyright $\odot 2022$ by author(s) and Scientific Research Publishing Inc. This work is licensed under the Creative Commons Attribution International License (CC BY 4.0).

http://creativecommons.org/licenses/by/4.0/

(c) (i) Open Access

\begin{abstract}
Introduction: The incidence of bladder cancer in women has steadily increased in recent years. The objective of our work is to study the epidemiological profile of bladder cancer in women in our population and the factors favoring its occurrence. Methods. This is a retrospective study carried out at the Mohamed Center VI for cancer treatment, involving 27 women during a two-year period from January 2019 to December 2020. We studied the epidemiological characteristics in these patients, the risk factors and the revealing symptoms. Results. The average age of patients was 67.5 years old and $66.66 \%$ of the patients were over 65 years old. One patient had in her antecedents a tumor of the upper excretory tract and 2 patients had an associated renal tumor. The notion of smoking was found in 3 patients. The time to treatment was less than 6 months in $44.4 \%$ of cases, between 6 and 12 months in $18.5 \%$ of cases, and more than a year in $37 \%$ of cases. The symptoms were marked by hematuria in $96.3 \%$ of cases, and irritative disorders in $81.4 \%$ of cases. In our series; the tumor was unifocal in $77.77 \%$ of the cases, and multifocal in the remainder of the cases. Urethrohydronephrosis was found in $74 \%$ of cases. Histologically, it was an urothelial carcinoma in $81.48 \%$ of cases. The tumor was locally advanced in $11.11 \%$ of cases, and metastatic in $11.11 \%$ of cases. Lymph node involvement was found in $33.33 \%$ of cases. $48.1 \%$ of cases underwent surgery. The operative procedure consisted of a partial cystectomy in $46.1 \%$ of cases, and only $18.5 \%$ was suitable for trimodal treatment. Conclusion: Few studies have addressed the epidemiology of bladder cancer in women due to the low incidence, but it is currently increasing steadily in recent years. These modifications are explained by the change in social habits in women.
\end{abstract}

\section{Keywords}

Bladder Carcinoma, Women, Epidemiology, Therapeutics Results 


\section{Introduction}

Bladder cancer occurs at an average age of 65 and the male/female ratio is estimated to be 4.8:1 [1]. However, the incidence of this disease in women has steadily increased in recent years.

Smoking is the main risk factor, and gross hematuria is the revealing symptom in more than $80 \%$ of cases.

The standard treatment is anterior pelvectomy in women; but given the morbidity and mortality associated with this surgery, as well as the deterioration in quality of life, patients must be selected for trimodal treatment.

We report here a series collected within the Mohamed VI center for cancer treatment; in order to study the epidemiological profile of bladder cancer in women in our population, the factors favoring its occurrence and its suggestive signs.

\section{Methods}

This is a retrospective study of twenty-seven patients followed for bladder cancer at the Mohamed VI Cancer Treatment Center. These patients were collected over a two-year period; January 2019 to December 2020.

Our hospital center uses a computerized system "Enova" to record all data concerning the patient and her pathology: identity, medical history, and initial clinical, paraclinical and anatomopathological assessment. It also contains the therapeutic decision after a multidisciplinary consultation meeting and the post-therapeutic results.

Epidemiological and therapeutic data were collected on an excel file from the computerized medical records of the patients followed regularly in consultation after the end of their treatment.

\section{Results}

Twenty-seven patients were collated. The average age was 67.5 years with extremes ranging from 45 to 100 years. $66.66 \%$ of the patients were over 65 years old.

3 patients in our series were smokers. $25.9 \%$ were diabetic, and $18.5 \%$ were followed for high blood pressure.

We found the notion of recurrent urinary tract infections (cystitis) in one patient. But no case of bilharzia has been detected. A patient was treated for an upper excretory tract tumor, two patients had an associated renal tumor, and no case of bladder or renal lithiasis was found in our series.

The time to treatment was less than 6 months in 44.4\% of cases, between 6 and 12 months in $18.5 \%$ of cases, and more than a year in $37 \%$ of cases. The symptoms were marked by hematuria in $96.3 \%$ of cases, and irritative disorders in $81.4 \%$ of cases.

The site of the tumor in order of frequency was as follows: the posterior wall in $37 \%$ of the cases, the latéro-left wall in $25.9 \%$ of the cases. The walls; anterior, 
latéro-right and the entire bladder were affected in $11.11 \%$ of cases for each of them.

In our series, the tumor was unifocal in $77.77 \%$ of the cases, and multifocal in the remainder of the cases. Urethrohydronephrosis was found in $74 \%$ of cases.

Histologically, it was an urothelial carcinoma in $81.48 \%$ of cases, of which the component of squamous and micropapillary carcinoma was found in $13.6 \%$ of cases. Other less frequent histological type's adenocarcinoma type in $7.4 \%$ of cases, muciparous kitten ring carcinoma in $3.7 \%$ of cases, and pure squamous cell carcinoma in $3.7 \%$ of cases.

The extension assessment made it possible to classify patients with locally advanced stage in $11.11 \%$ of cases, and metastatic in $11.11 \%$ of cases. Lymph node involvement was found in $33.33 \%$ of cases.

$48.1 \%$ of cases underwent surgery. The operative procedure consisted of partial cystectomy in $46.1 \%$ of cases, total cystectomy with ureterostomy in $38.5 \%$ of cases, and total cystectomy with neo-bladder in $15.4 \%$ of cases. Just $30.7 \%$ of patients received neoadjuvant chemotherapy during surgery. However, all the operated patients; received adjuvant chemotherapy-type treatment in $92.3 \%$ of cases and radiotherapy in one patient given the R1 resection of the tumor (Figure 1 and Figure 2).

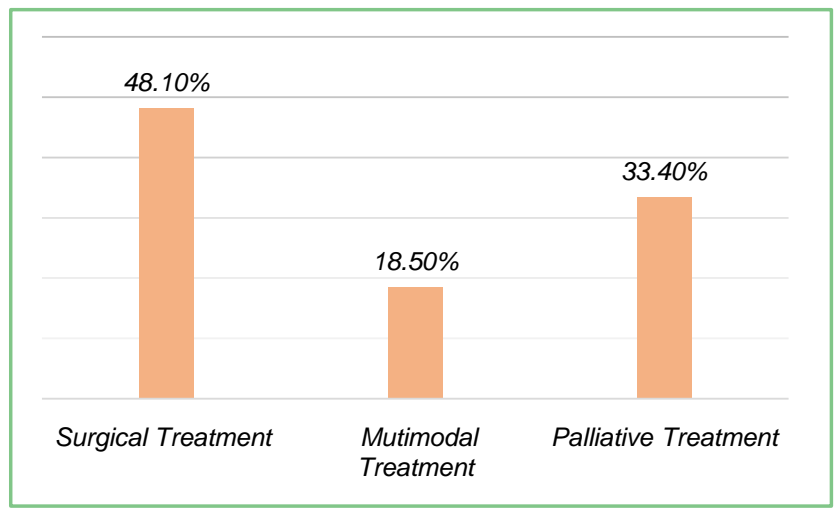

Figure 1. Therapeutic modalities.

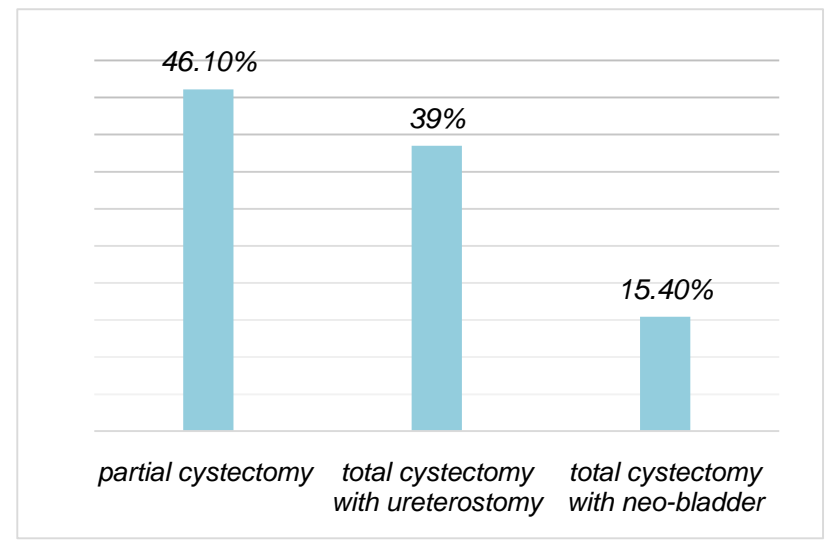

Figure 2. Surgical treatment. 
In our study population, only $18.5 \%$ were suitable for trimodal treatment (Figure 1).

The follow-up is based on clinical examination, thoraco-abdomino-pelvic scanner and cystoscopy in case of trimodal treatment every 3 - 4 months during the first year and every 6 months during the second and third year.

Our average follow-up was 25 months, a relatively low follow-up linked to a large number of lost to follow-up in $40.7 \%$ of cases. However; $29.6 \%$ are progressing, $18.5 \%$ are in remission, and only one death according to the available data.

\section{Discussion}

The incidence of bladder tumors in women is increasing worldwide.

Smoking is the most important factor [2]. In fact, the risk of developing bladder cancer is 2 to 4 times higher in smokers compared to non-smokers and this risk increases with the intensity and/or duration of smoking [3] [4]. In our series, $11.11 \%$ of patients were exposed to tobacco.

Environmental factors have been associated with an increased risk of developing bladder cancer; to know; drinking water disinfection techniques using chlorine with a relative risk of 1.7 to 3.1 with respect to bladder cancer [5]. Exposure to urothelial carcinogens in particular; to aromatic amines [6] [7], as well as to polycyclic aromatic hydrocarbons [8], whether recent or old.

McGrath and colleagues (2006) reported an increased risk of bladder cancer in postmenopausal women $(\mathrm{p}=0.30)$. Hormone replacement therapy during menopause and late menopause at age 48 or older has been shown to protect against bladder cancer [9] [10].

Other authors suggest that the prevalence of pathologies affecting bladder emptying increases with age such as genital prolapse in women [11]. Causes chronic urinary stasis which is associated with an increase in the contact time of carcinogens with the urothélium, again promoting its carcinogenesis [12].

Hematuria is the main sign of bladder cancer and is often present. But we noticed that our elderly patients had longer diagnostic times; and this due according to studies the increase in the prevalence of hematuria secondary to taking antiplatelet agents or anticoagulants according to Bhatt et al. in the elderly population [13], and the underestimation of hematuria given the frequency of benign causes of hematuria which increases with age, mainly concerning genital prolapse in women [11] and urinary tract infections [14]. What led to American college of physicians to recommend no longer interpret hematuria as a consequence of antiaggregant or anticoagulant treatment but systematically look for a cause, in particular tumor [15].

The standard treatment for muscle-infiltrating urothelial bladder tumors is based on neoadjuvant chemotherapy based on platinum salts followed by anterior pelvectomy in women with evidently ilio-obturator lymph node dissection [16]. It is a heavy and complex surgery, in particular in elderly patients and/or 
suffering from significant associated diseases. The morbidity and mortality associated with this intervention remains high, with a complication rate between $40 \%$ and $60 \%$ and a mortality rate of $9 \%$ in the first three months postoperatively [17] [18]. In addition, patients who have had a cystectomy suffer from an impaired quality of life regardless of the mode of urinary diversion, both urinary, digestive or sexual [19].

However; phase II pilot trials validated trimodal treatment in selected patients; combining the most complete possible transurethral resection of the tumor followed by concomitant chemoradiotherapy. Radiotherapy was administered up to a dose level of 40 to $45 \mathrm{~Gy}$, with concomitant cisplatin in various modalities, with or without 5-fluorouracil, followed 5 to 6 weeks later by cystoscopy with biopsies control of the tumor bed. Patients in complete histological remission received additional chemoradiotherapy up to a total dose of 55 to 65 Gy. In contrast; a cystectomy was offered to patients with a tumor residue [20] [21].

After trimodal treatment; 5-year bladder retention rates were between 40 and $80 \%$ in the recent series [22] [23] [24]. As well as currently, we have recent meta-analyses and reviews of the literature which did not show a significant difference in terms of survival between the concomitant chemoradiotherapy arm and the surgery arm with a $p=0.32$ [25] [26].

\section{Conclusions}

Few studies have addressed the epidemiology of bladder cancer in women due to the low incidence, but it is currently increasing steadily in recent years.

The advanced age of onset and the frequency of etiologies of benign hematuria in elderly women explain the late diagnosis in this population, hence the interest in systematizing cystoscopy.

\section{Conflicts of Interest}

The authors declare no conflicts of interest regarding the publication of this paper.

\section{References}

[1] Mishriki, S.F., Vint, R. and Somani, B.K. (2012) Half of Visible and Half of Recurrent Visible Hematuria Cases Have Underlying Pathology: Prospective Large Cohort Study with Long-Term Follow-Up. The Journal of Urology, 187, 1561-1565. https://doi.org/10.1016/i.juro.2011.12.100

[2] Pelucchi, C., La Vecchia, C., Negri, E., Dal Maso, L. and Franceschi, S. (2002) Smoking and Other Risk Factors for Bladder Cancer in Women. Preventive Medicine, 35, 114-120. https://doi.org/10.1006/pmed.2002.1061

[3] Kirkali, Z., Chan, T., Manoharan, M., et al. (2005) Bladder Cancer: Epidemiology, Staging and Grading, and Diagnosis. Urology, 66, 4-34. https://doi.org/10.1016/j.urology.2005.07.062

[4] Silverman, D.T., Devesa, S.S., Moore, L.E., et al. (2006) Bladder Cancer. In: 
Schot-Tenfeld, D. and Fraumeni, J., Eds., Cancer Epidemiology and Prevention, Oxford University Press, New York, 1101-1127. https://doi.org/10.1093/acprof:oso/9780195149616.003.0058

[5] Boorman, G.A. (1999) Drinking Water Disinfection Byproducts: Review and Approach to Toxicity Evaluation. Environmental Health Perspectives, 107, 207-217. https://doi.org/10.1289/ehp.99107s1207

[6] IARC Working Group on the Evaluation of Carcinogenic Risks to Humans (1987) Overall Evaluations of Carcinogenicity: An Updating of IARC Monographs Volumes 1 to 42. International Agency for Research on Cancer, Lyon.

[7] Pop, W., Schmieding, W., Speck, M., Vahrenholz, C. and Norpoth, K. (1992) Incidence of Bladder Cancer in a Cohort of Workers Exposed to 4-Chloro-o-Toluidine While Synthesizing Chlordimeform. British Journal of Industrial Medicine, 49, 529-531. https://doi.org/10.1136/oem.49.8.529

[8] Boffetta, P., Jourenkova, N. and Gustavsson, P. (1997) Cancer Risk from Occupational and Environmental Exposure to Polycyclic Aromatic Hydrocarbons. Cancer Causes \& Control, 8, 444-472. https://doi.org/10.1023/A:1018465507029

[9] McGrath, M., Michaud, D.S. and De Vivo, I. (2006) Hormonal and Reproductive Factors and the Risk of Bladder Cancer in Women. American Journal of Epidemiology, 163, 236-244. https://doi.org/10.1093/aje/kwj028

[10] Prizment, A.E., Anderson, K.E., Harlow, B.L. and Folsom, A.R. (2007) Reproductive Risk Factors for Incident Bladder Cancer: Iowa Women's Health Study. International Journal of Cancer, 120, 1093-1098. https://doi.org/10.1002/ijc.22418

[11] Pillalamarri, N., Shalom, D., Sanidad, S., Akerman, M., Lind, L. and Winkler, H. (2015) The Prevalence of Microscopic Hematuria in a Cohort of Women with Pelvic Organ Prolapse. International Urogynecology Journal, 26, 85-90. https://doi.org/10.1007/s00192-014-2450-6

[12] Birnbaum, L.S. (1987) Age-Related Changes in Carcinogen Metabolism. Journal of the American Geriatrics Society, 35, 51-60. https://doi.org/10.1111/j.1532-5415.1987.tb01319.x

[13] Bhatt, N.R., Davis, N.F., Nolan, W.J., Flynn, R.J., McDermott, T., Thomas, A.Z. and Manecksha, R.P. (2018) Incidence of Visible Hematuria among Antithrombotic Agents: A Systematic Review of over 175,000 Patients. Urology, 114, 27-32. https://doi.org/10.1016/j.urology.2017.11.023

[14] Tandogdu, Z. and Wagenlehner, F.M. (2016) Global Epidemiology of Urinary Tract Infections. Current Opinion in Infectious Diseases, 29, 73-79. https://doi.org/10.1097/QCO.0000000000000228

[15] Nielsen, M. and Qaseem, A. (2016) Hematuria as a Marker of Occult Urinary Tract Cancer: Advice for High-Value Care from the American College of Physicians. Annals of Internal Medicine, 164, 488-497. https://doi.org/10.7326/M15-1496

[16] Rouprêt, M., Neuzillet, Y., Pignot, G., Compérat, E., Audenet, F., Houédé, N., et al. (2018) French ccAFU Guidelines-Update 2018-2020: Bladder Tumors. Progrès en Urologie, 28, S46-S78. https://doi.org/10.1016/j.purol.2018.07.283

[17] Froehner, M., Brausi, M.A., Herr, H.W., Muto, G. and Studer, U.E. (2009) Complications Following Radical Cystectomy for Bladder Cancer in the Elderly. European Urology, 56, 443-454. https://doi.org/10.1016/j.eururo.2009.05.008

[18] Aziz, A., et al. (2014) Prediction of 90-Day Mortality after Radical Cystectomy for Bladder Cancer in a Prospective European Multicenter Cohort. European Urology, 66, 156-163. https://doi.org/10.1016/j.eururo.2013.12.018 
[19] Ziouziou, I., et al. (2018) Ileal Conduit vs Orthotopic Neobladder: Which One Offers the Best Health-Related Quality of Life in Patients Undergoing Radical Cystectomy? A Systematic Review of Literature and Meta-Analysis. Progrès en Urologie, 28, 241-250. https://doi.org/10.1016/j.purol.2018.02.001

[20] Tester, W., Porter, A., Asbell, S., Coughlin, C., Heaney, J., Krall, J., et al. (1993) Combined Modality Program with Possible Organ Preservation for Invasive Bladder Carcinoma: Results of RTOG Protocol 85-12. International Journal of Radiation Oncology, Biology, Physics, 25, 783-790. https://doi.org/10.1016/0360-3016(93)90306-G

[21] Rotman, M., Aziz, H., Porrazzo, M., Choi, K.N., Silverstein, M., Rosenthal, J., et al. (1990) Treatment of Advanced Transitional Cell Carcinoma of the Bladder with Irradiation and Concomitant 5-Fluorouracil Infusion. International Journal of Radiation Oncology, Biology, Physics, 18, 1131-1137. https://doi.org/10.1016/0360-3016(90)90449-T

[22] Rödel, C., Grabenbauer, G.G., Kühn, R., Papadopoulos, T., Dunst, J., Meyer, M., et al. (2002) Combined-Modality Treatment and Selective Organ Preservation in Invasive Bladder Cancer: Long-Term Results. Journal of Clinical Oncology, 20, 3061-3071. https://doi.org/10.1200/JCO.2002.11.027

[23] Mak, R.H., Hunt, D., Shipley, W.U., Efstathiou, J.A., Tester, W.J., Hagan, M.P., et al. (2014) Long-Term Outcomes in Patients with Muscle-Invasive Bladder Cancer after Selective Bladder-Preserving Combined-Modality Therapy: A Pooled Analysis of Radiation Therapy Oncology Group Protocols 8802, 8903, 9506, 9706, 9906, and 0233. Journal of Clinical Oncology, 32, 3801-3809. https://doi.org/10.1200/JCO.2014.57.5548

[24] Nagao, K., Hara, T., Nishijima, J., Shimizu, K., Fujii, N., Kobayashi, K., et al. (2017) The Efficacy of Trimodal Chemoradiotherapy with Cisplatin as a Bladder-Preserving Strategy for the Treatment of Muscle-Invasive Bladder Cancer. Urologia Internationalis, 99, 446-452. https://doi.org/10.1159/000477912

[25] Arcangeli, G., Strigari, L. and Arcangeli, S. (2015) Radical Cystectomy versus Organ-Sparing Trimodality Treatment in Muscle-Invasive Bladder Cancer: A Systematic Review of Clinical trials. Critical Reviews in Oncology/ Hematology, 95, 387-396. https://doi.org/10.1016/j.critrevonc.2015.04.006

[26] Fahmy, O., Khairul-Asri, M.G., Schubert, T., Renninger, M., Malek, R., Kübler, H., et al. (2018) A Systematic Review and Meta-Analysis on the Oncological Long-Term Outcomes after Trimodality Therapy and Radical Cystectomy with or without neoadjvant Chemotherapy for Muscle Invasive Bladder Cancer. Urologic Oncology: Seminars and Original Investigations, 36, 43-53. https://doi.org/10.1016/j.urolonc.2017.10.002 